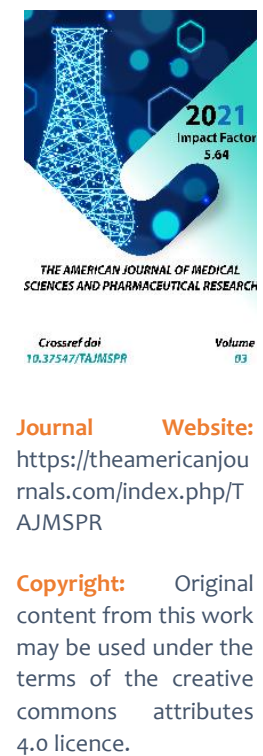

\section{Application Of Decompression Treatment For Mandibular Radicular Cysts}

\author{
M.A. Askarov
}

Assistant Of The Department Of Maxillofacial Region Diseases And Injuries, Tashkent State Institute Of Dentistry, Tashkent, Uzbekistan

\section{A.M. Azimov}

Candidate Of Medical Sciences, Associate Professor Of The Department Of Surgical Stomatology And Dental Implantology, Tashkent State Dentistry Institute, Tashkent, Uzbekistan

\section{Sh.K. Isokjonov}

Assistant Of The Department Of Maxillofacial Region Diseases And Traumas, Tashkent State Dentistry Institute, Tashkent, Uzbekistan

\title{
ABSTRACT
}

This article presents a clinical case of the treatment of a mandibular radicular cyst from 3.6 teeth by creating a decompression "window", which allows the cyst volume to be reduced up to complete restoration of the bone structure as well as ensuring the integrity of the surrounding anatomical structures. The subsequent filling of the cavity with newly formed bone is due to secondary osteogenesis. This operation can be performed on an outpatient basis in a dental surgery room under local anesthesia

\section{KEYWORDS}

Decompression method, radicular cyst, lower jaw.

\section{INTRODUCTION}

To date, one of the most pressing problems of modern maxillofacial surgery is the treatment of human jaw cysts a traumatically and with preservation of the integrity of the dentition. The significance of this topic is determined by its frequency, prevalence among children, adolescents and young adults. Patients with odontogenic cysts of the jaw occupy an important place in the structure of dental diseases. [10].
Radicular cysts account for $94-96 \%$ of odontogenic cysts of the jaws detected in adults. The most frequent localization of radicular cysts is on the upper jaw, less often on the lower jaw $[3,4,6]$. Despite modern conservative methods of treatment, the need for surgical treatment of odontogenic cysts has not decreased. The main surgical method for the treatment of odontogenic cysts of the jaw is cystectomy, and less frequently, cystotomy 
$[1,4,7]$. The results of scientific research indicate that the damage caused to the supporting dental tissues in youth is irreparable, and in middle age it leads to a significant destruction of the dental apparatus. Preservation of teeth located in the cyst area is the task of the dentist in the treatment of periradicular cysts $[2,3,4]$. It is important to make a correct diagnosis, excluding oral oncological pathology because of the similarity of the clinical manifestations of the pathology $[5,8]$. For the treatment of radicular cysts, classical elective surgical interventions (cystotomy, cystoectomy) have long been used, with the help of which a high cure rate is achieved. The choice of treatment method depends on the size of the cyst, proximity to important anatomical structures, and location in the jaw $[1,4,7]$. Surgery can be performed both in outpatient conditions and in the hospital. However, this can be achieved under certain conditions (removal of the "causal" teeth, radicality of the operation). In modern conditions, when patients have requirements for a fast rehabilitation process and preservation of aesthetics throughout the entire treatment period, some modifications to the classic stages of surgery are required. Treatment of cysts begins with diagnosis. Computed tomography is a mandatory step in the examination. The second important point is the morphological examination of the mass. In some cases with classic slow growth, typical location of cysts, one-stage removal of the mass and replacement of the formed defect with autologous, xenogeneic or allogenic graft after morphological examination is acceptable [9].

Purpose of the study: To substantiate the value of using the decompression method for the treatment of mandibular radicular cysts.

\section{MATERIALS AND METHODS}

Patient M., 14 years old, in October 2020 went to a private dental clinic "Azimov brothers". From the medical history it is known that in 2018, the thirty-sixth tooth was treated for caries, at the time of treatment she had no active complaints. In 2019, noted "flux" in the projection of the root of the 36th tooth and enlarged submandibular lymph nodes. She did not seek medical attention. In 2020, she went to the Department of Pediatric Maxillofacial Surgery, where she was diagnosed with "Radicular cyst of the lower jaw from teeth 3.6, 3.7" and a CT scan of the maxillofacial region was performed. The findings revealed a nidus of bone destruction in the lateral region of the lower jaw in the region of 3.6, 3.7, 3.8 (Fig. 1, 2). After examination by a dental surgeon, the diagnosis was: Radicular cyst of the lower jaw from tooth 36 . The patient was offered operative treatment.

\section{RESULTS AND DISCUSSION}

A clinical and laboratory examination was performed in preparation for surgery. As a result, no deformities of the lower jaw were detected, palpation of the frontal region of the lower jaw revealed no evidence of neoplasm in the bone thickness, the cyst wall did not sag when pressing on it, the mucosa in the cyst projection was not discolored, pale pink in color, moderately moistened. There were no visible external changes of the maxillary system. An electroexcitation study of the teeth showed no changes in the pulp of the 37th tooth (EOD $2 \mathrm{~mA}$ ). Percussion of teeth 36, 37 was slightly painful, Fries-test of teeth 3.6, 3.7 was negative, Drill-test of tooth 37 was positive. It was decided to perform a decompression type cystotomy of the lower jaw, creating a trepanation hole. This method 
was chosen in order to completely remove the visible lesion, minimize the risk of recurrence and achieve an optimum aesthetic and functional result. After a detailed explanation to the patient and her parents about the upcoming surgery, they agreed to undergo the surgery and signed an informed consent form. The patient was referred to a general dentist for endodontic treatment of 3.6 teeth.

Under local infiltration anaesthesia Sol. Articaini $1: 100000-1.5 \mathrm{ml}$ a $0.5 \mathrm{~cm}$ vertical soft tissue incision was made in the apex projection of the distal root of the 3.6 tooth. Skeletonisation of the compact lamina of the mandibular frontal region was performed. No visual bone changes (patterns, deformities) were detected. The bone cavity was opened using a ball cutter. The diameter of the trepanation hole was $0.5 \mathrm{~cm}$. About $5 \mathrm{ml}$ of clear, light yellow fluid was evacuated from the bone cavity under pressure. The fluid was taken for cytological examination. A catheter was placed and the bone cavity was flushed with warm physiological solution to remove cleavage products and enzymes. Hemostasis control is carried out. The wound was sutured with 6-0 polyamide thread. (Figure 3) On the following three days, the cavity was irrigated with warm saline solution. There was no discharge during the lavage procedure. The catheter was removed on the fourth day.

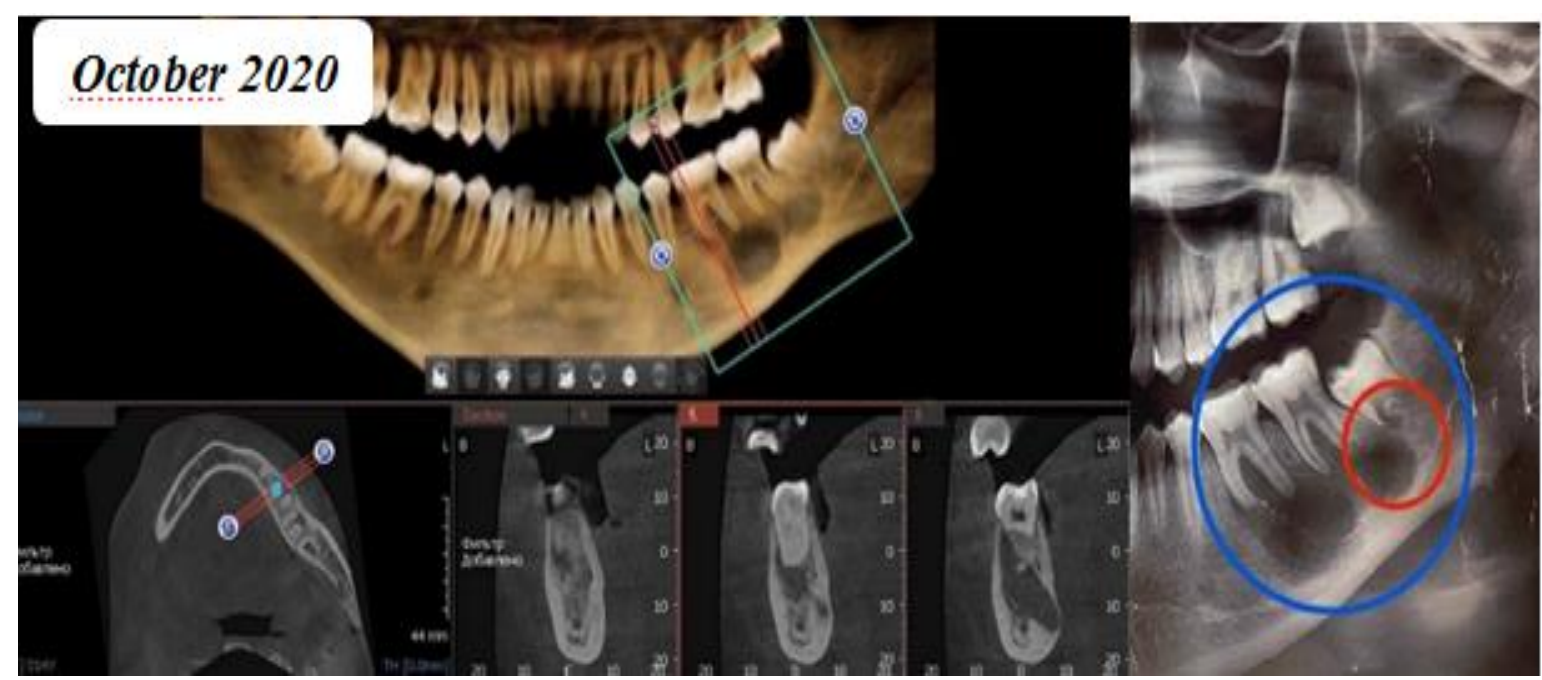

Fig.1. Computed tomography scan of the patient before treatment. 


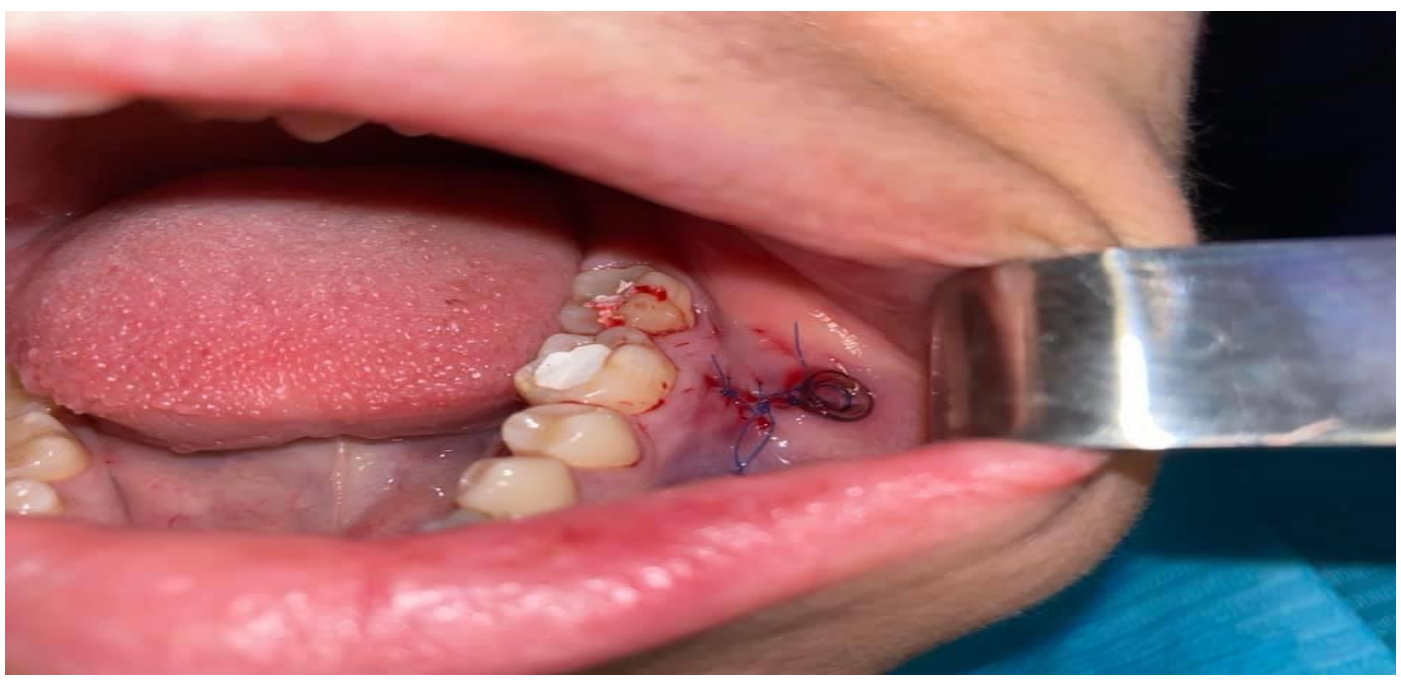

Fig. 2. After inserting the catheter and suturing.

The early postoperative period was smooth. Wound healing proceeded by primary tension without complications. The period of disability was 1 week. A trepanation hole was formed to create a decompression effect, allowing treatment of the cyst cavity with antiseptic solutions.

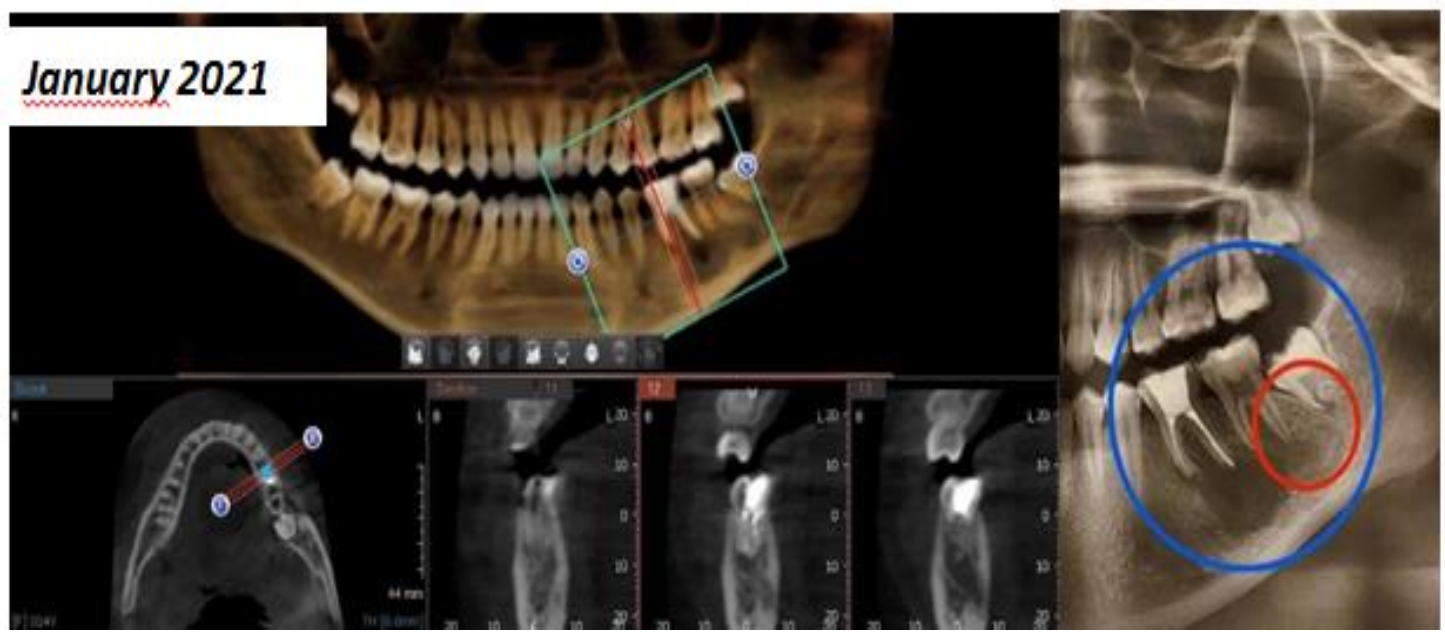

Fig. 3. Computed tomography scan of a patient 6 months after treatment

Follow-up examinations after 3 and 6 months using radiotherapy showed gradual filling of the defect with newly formed bone tissue, which over time began to acquire normal density (Fig. 3). A follow-up examination after 6 months showed satisfactory results with no recurrence. 


\section{CONCLUSIONS}

Radicular cysts of the jaw are a common pathology among young adults, depriving children and young adults to lead a normal life. Based on the performed conservative treatment technique with the creation of the "window" allows to gradually reduce the volume of the cyst until the complete restoration of the bone structure, while ensuring the integrity of the surrounding structures (teeth, neurovascular bundle of the third branch of the trigeminal nerve). Effective cyst decompression, which is achieved by creating a "window" in the bone cavity allows not only to sanitise the cyst cavity with antiseptic solutions, but also to obtain the necessary histological material (bone material and cyst membrane) for further examinations. The subsequent filling of the bone cavity defect with newly formed bone tissue is due to secondary osteogenesis. The operation can be carried out in a dental surgery under local anaesthetic. The period of incapacity for work is usually less than 1 week.

\section{REFERENCES}

1. Gubaidullina, E. Ya., Tsegelnik, L. N., Luzina, V. V., \& Topleninova, D. Yu. (2007). Experience in the treatment of patients with extensive jaw cysts. Dentistry, 86 (3), 51-54.

2. Eshiev, A.M. (2011). Methods of treating radicular cysts with various osteoplastic agents. Young scientist. 8 (31). pp. 149-152.

3. Eshiev, A.M., \& Eshiev, D.A. (2012). Stimulation of the healing of postoperative bone defects on the alveolar processes of the upper and lower jaw. Young Scientist, (3), 445447.
4. Kuzminykh, I.A. (2008). Surgical treatment of radicular cysts using the biocomposite material "Allomatriximplant" and platelet-rich fibrin (Doctoral dissertation, Abstract of the thesis ... can. Of medical sciences).

5. Mamaeva, E.V. (2007). Periodontal status and functional state of the body in adolescents (Doctoral dissertation, Central Research Institute of Dentistry, Ministry of Health of the Russian Federation). p.196.

6. Raad, Z.K., Veselova, T.V., \& Biabi, A. (2014). Osteoplastic replacement of jaw defects in the treatment of odontogenic cysts. Institute of Dentistry, (2), 36-39.

7. Rakhimov, Z.K., Chirgaliev, M. Zh., \& Pulatova, Sh.K. (2019). Improvement of methods of treatment of radicular cysts of the jaws. Biology and Integrative Medicine, (2 (30)).

8. Zinecker, D.A. (2013). Features of chronic hypertrophic gingivitis in adolescents 13-15 years old (Doctoral dissertation, Kazan State Medical University).

9. Shomurodov. K.E. (2010). Features of cytokine balance in gingival fluid at odontogenicphlegmon of maxillofacial area. Doctor-aspirant. 42(5.1) pp.187192.

10. Shomurodov, K. E. (2020). Comparative evaluation the anatomical and functional state of the. Journal of research in health science, 1(4), 54-57.

11. Djuraeva, D. D., \& Berdiyeva, Z. M. (2016). Cultural heritage as a factor of human development (on the example of Uzbekistan). Ученый XXI века, 23. 